\title{
An analog front-end enables electrical impedance spectroscopy system on-chip for biomedical applications
}

\author{
Fernando Seoane $^{1,2}$, Javier Ferreira ${ }^{1}$, Juan José Sanchéz ${ }^{1}$ \\ and Ramon Bragós ${ }^{3}$ \\ ${ }^{1}$ School of Engineering, Medical Electronics Group, University College of Borås, Borås 50190, \\ Sweden \\ ${ }^{2}$ Department of Signals and Systems, Division of Biomedical Engineering, Chalmers University \\ of Technology, Gothenburg 41296, Sweden \\ ${ }^{3}$ Department of Electronic Engineering, Technical University of Catalonia, Barcelona 08034, \\ Spain \\ E-mail: fernando.seoane@hb.se
}

Received 30 November 2007, accepted for publication 12 May 2008

Published 11 June 2008

Online at stacks.iop.org/PM/29/S267

\begin{abstract}
The increasing number of applications of electrical bioimpedance measurements in biomedical practice, together with continuous advances in textile technology, has encouraged several researchers to make the first attempts to develop portable, even wearable, electrical bioimpedance measurement systems. The main target of these systems is personal and home monitoring. Analog Devices has made available AD5933, a new system-onchip fully integrated electrical impedance spectrometer, which might allow the implementation of minimum-size instrumentation for electrical bioimpedance measurements. However, AD5933 as such is not suitable for most applications of electrical bioimpedance. In this work, we present a relatively simple analog front-end that adapts AD5933 to a four-electrode strategy, allowing its use in biomedical applications for the first time. The resulting impedance measurements exhibit a very good performance in aspects like load dynamic range and accuracy. This type of minimum-size, system-on-chip-based bioimpedance measurement system would lead researchers to develop and implement light and wearable electrical bioimpedance systems for home and personal health monitoring applications, a new and huge niche for medical technology development.
\end{abstract}

Keywords: electrical bioimpedance spectroscopy, system-on-chip, fourelectrode method

(Some figures in this article are in colour only in the electronic version) 


\section{Introduction}

The continuous progress of medical applications of electrical bioimpedance spectroscopy (EBIS) (Aberg et al 2005, Moissl et al 2006, Caduff et al 2006), combined with recent advances in textile electrode technology (Pacelli et al 2006, Paradiso and De Rossi 2006), enable the development of bioimpedance-based measurement systems for home monitoring (Medrano et al 2007, Vuorela et al 2007) and personal monitoring (Scheffler et al 2003).

Traditionally, wearable systems for on-body measurements have focused on the acquisition of biosignals and activity monitoring, in the line of the EU project Wealthy IST-2001-37778 (Paradiso et al 2004). The European Commission has specifically supported several textile-based healthcare projects, e.g. BIOTEX IST-2004-016789, CONTEXT IST2004-027291, MERMOTH FP6-IST-508272, MyHeart IST-2002-507816, OFSETH IST2005-027869, PROETEX IST-2004-026987 and STELLA FP6-IST-028086. Currently, it is possible to find commercial products for personal healthcare monitoring, e.g. Lifeshirt ${ }^{\circledR}$ of Vivometrics ${ }^{\circledR}$ and adiSTAR ${ }^{\circledR}$ of Adidas.

Often the acquired signals are ECG and heart rate with electrodes, movement with accelerometers and respiration rate with piezoelectric and/or inductive sensors. EBI has not been among the typical measurements for monitoring until recently. Initially, EBI measurements target the respiration activity rate by impedance pneumography (Paradiso and De Rossi 2006, Seppä et al 2007), heart failure by impedance cardiography (Amft and Habetha 2007) and even body composition by bioelectrical impedance analysis (Hännikäinen et al 2007). Nowadays it is possible to find a commercially available, single-frequency ambulatory bioimpedance monitor for cardiac assessment: AIM-8 manufactured by BioImpedance Technology, Inc.

A concise review of wearable systems for physiological measurements can be found in Hännikäinen et al (2007). For comprehensive and detailed information about healthcare applications of smart textiles, see Van Langenhove (2007).

AD5933 is the first commercially available impedance network analyzer implemented in a single integrated circuit, and it allows us to take an important step from portable to wearable applications. The complexity of the impedance measurement system is reduced to basically one integrated circuit plus additional analog circuitry for signal conditioning, to meet the requirement for electrical bioimpedance (EBI) applications.

AD5933 alone is not suitable for EBI measurements on patients, or for several EBI applications, due to several factors, e.g. dc excitation, a voltage-driven system with large output impedance and a two-electrode system. In this work, we propose and validate the addition of an analog front-end (AFE) to fully adapt the AD5933 circuit to a fourelectrode strategy. In this way, the deflection signal is adapted for on-body measurements. The resulting system is a multifrequency measurement system with very few ICs which is suitable for wearable applications.

\section{Methodology}

\subsection{Materials}

The core of the measurement system is the impedance network converter integrated circuit AD5933. The impedance measurement system by itself is the evaluation board for the AD5933 circuit provided by Analog Devices Inc., the EVAL-5933EB ${ }^{4}$.

4 See www.analog.com. 
The AFE is implemented with general application op-amp circuits, instrumentation amplifiers and voltage references. An adaptation circuit for measurements of small impedance has been used as recommended by Analog Devices.

The software used to control AD5993 and display the impedance measurements is provided with the evaluation board. This software application has been adapted for correct four-electrode operation with Visual Basic 6.0.

All the documentation related to the AD5933 evaluation board, schematics, user manual and software is available at the Analog Devices Inc. website.

\subsection{Accuracy test}

Impedance measurements have been performed on pure resistive loads as well as on a $2 \mathrm{R}$ $1 \mathrm{C}$ series circuit; note the absence of physical electrodes or any equivalent dummy. The measurements have been done with and without the AFE in the frequency range from $5 \mathrm{kHz}$ to $100 \mathrm{kHz}$.

To study the error performance and its dependence on the load dynamic range, a pure resistive load has been measured at $10 \mathrm{kHz}$ and its deviation from the theoretical value has been calculated. The value of the working load has been changed from $50 \Omega$ to $1.6 \mathrm{k} \Omega$.

To study the frequency performance of the measurement system, the $2 \mathrm{R}-1 \mathrm{C}$ series circuit has been used as a measurement load, measuring both resistance and reactance.

\subsection{Four-electrode validation}

To validate the proper implementation of the four-electrode technique, impedance measurements have been performed on a $2 \mathrm{R}-1 \mathrm{C}$ series circuit phantom. The measurements have been done by modeling the electrodes with an equivalent electrical circuit. The electrodeequivalent circuits have been connected in series with the phantom and the four leads, for both injection and measurement. The elements and values of the equivalent circuit have been selected to model the effect of the electrode polarization impedance, $Z_{\mathrm{EP}}$.

\section{Electrical bioimpedance instrumentation}

Electrical bioimpedance measurements are most often performed as deflection measurements by measuring the response of the system to an external electrical excitation (Pallàs-Areny and Webster 2001). Current is injected in the tissue under study (TUS) and the voltage drop caused in the load is measured or, reciprocally, the voltage is applied to the TUS and the correspondingly caused current through the TUS is measured. In either case, a generator for the excitation signal is required as well as a sensing stage to measure the response of the TUS.

Once the response to the excitation in voltage or current is measured by the system, the next step in an impedance measurement system is the impedance estimation process. This functional block estimates the complex impedance from the electrical measurements obtained, and there are several approaches to implementing this function. Sine correlation and Fourier analysis are choices often selected.

The electrodes are a critical element of an EBI measurement system; they function as an electronic-to-ionic interface between the electronic conductor in the measurement leads and the ionic conductor in the load, i.e. biological tissue. This interface can be described as a parallel circuit of a variable resistance $R_{\mathrm{E}}$ and a variable capacitance $C_{\mathrm{E}}$; in addition, there is also a variable voltage source $U_{\mathrm{EP}}$ at the interface, as depicted in figure 1 . The electrode impedance as well as the skin-electrode contact impedance can influence the impedance 


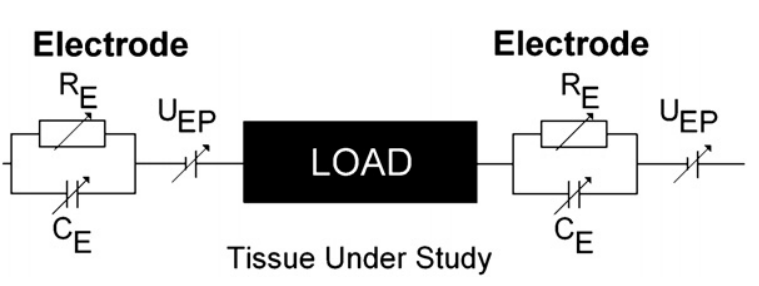

Figure 1. Equivalent model for the electrical interface, ionic-electronic, responsible for the electrode impedance $Z_{\mathrm{EP}}$ and its connection with the working load.

measurement to a very great extent, especially when the measurement setup is implemented as a two-electrode system.

In the two-electrode method, the same pair of electrodes is used to excite and to measure the response of the tissue. Therefore the impedance of the electrode, see figure 1, will be added to the impedance of the load. For instance if current is injected and voltage is measured, then the voltage measurement will contain the voltage drop caused in the TUS as well as the voltage drop caused in $C_{\mathrm{E}}$ and $R_{\mathrm{E}}$.

The four-electrode method is a robust electrode setup that reduces the influence of the electrode impedance and the skin-electrode contact impedance. This method uses a pair of electrodes to excite the TUS and a different pair of electrodes to measure the response. In the case of current excitation, electrical current does not flow through the sensing electrodes because of the high input impedance of the differential amplifiers. Therefore, the sensed voltage does not contain any voltage drop caused by $C_{\mathrm{E}}$ and $R_{\mathrm{E}}$ (Pallàs-Areny and Webster 2001).

\subsection{AD5933 impedance converter}

AD5933 is a two-electrode impedance measurement system, with a large dynamic range of the measurement load. According to the datasheet, AD5933 is able to measure loads ranging from $1 \mathrm{k} \Omega$ to $1 \mathrm{M} \Omega$, although the auxiliary resistor connected to the input feedback resistor (RFB) should be tailored for the specific range.

The AD5933 integrated circuit contains all the necessary elements to implement a fully integrated impedance spectrometer-in this case, a waveform generator, a voltage source output, a current measurement input, a Fourier-based impedance estimator and even a serial communication port. See figure 2 .

Note that the impedance estimation method is based on the Fourier analysis decomposition of both the reference signal and the measurement signal. In this case the reference signal represents the applied voltage, while the measurement signal represents the current through the measurement load. The DFT block provides the result as the ratio of reference over measurement, i.e. volts over amperes, thus impedance in $\Omega$, as indicated in (1). In this manner, the REAL and IMAGINARY registers contain resistance and reactance information, respectively:

$$
\frac{\text { REFERENCE SIGNAL }}{\text { MEASUREMENT SIGNAL }}=\frac{V_{\mathrm{OUT}}(\omega)}{I_{\mathrm{IN}}(\omega)}=Z(\omega) \quad(\Omega) \text {. }
$$




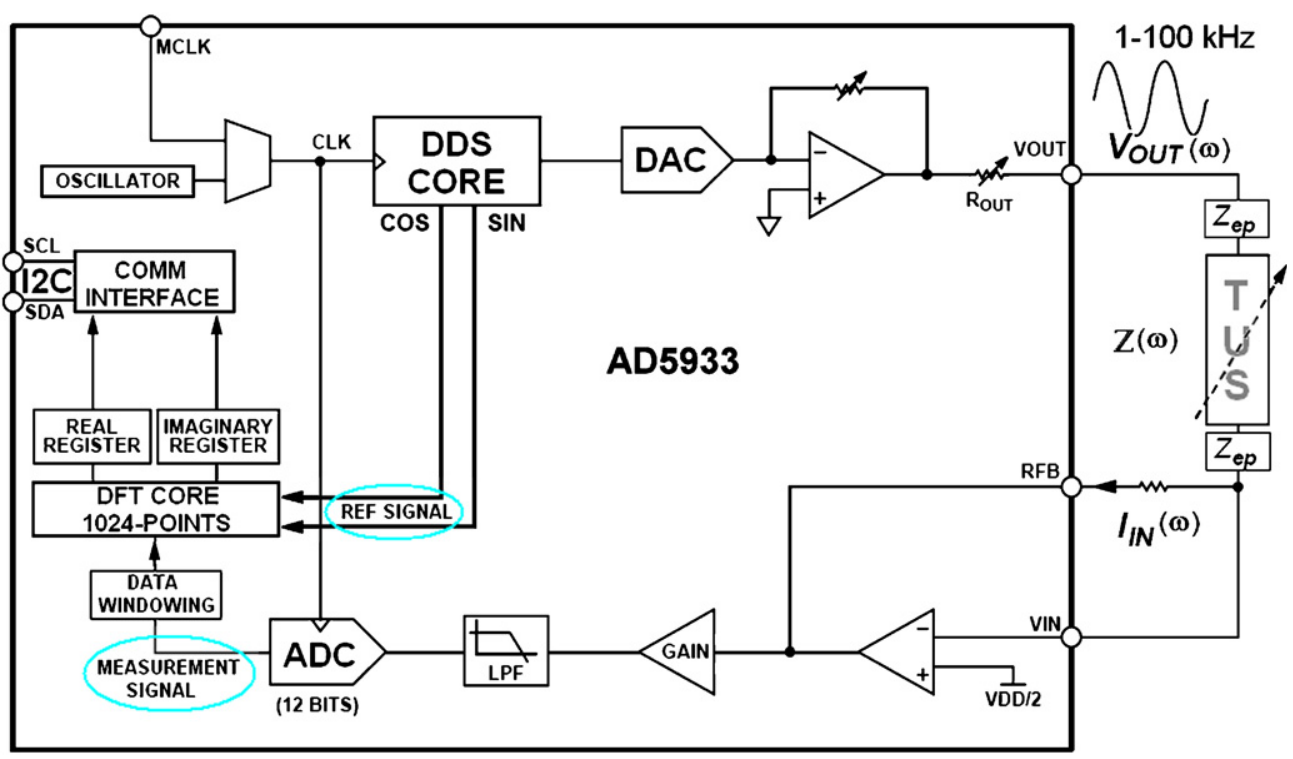

Figure 2. The functional block diagram of AD5933 for measurements of bioimpedance.

\subsection{AD5933 as a bioimpedance device}

The first point to note is that the AD5933 circuit is a two-electrode impedance measurement device. This fact by itself limits severely the range of application of usage, e.g. applications of spectral characterization are basically discarded since the impedance measurement obtained will also contain the electrode polarization impedance as well as the electrode-skin impedance.

Another important limitation of AD5933 is a safety issue: the voltage output contains a dc level, dc $c_{\text {bias }}$, which is different from the dc level at $V_{\text {in }}$, namely $V_{\mathrm{dd} / 2}$. This imbalance produces a dc voltage across the electrodes and the TUS, introducing dc current in the TUS, which can be a health hazard for the patient.

Moreover, AD5933 is a voltage-driven measurement system without any control over the injected current. This might be a safety hazard issue since the injected current can be larger than the limits set by IEC-60601.

Most often, values of EBI measurements fall within the range of tens or hundreds of Ohms than in the range of thousands of ohms. According to the specifications in the datasheet, the lower limit of AD5933 is $1 \mathrm{k} \Omega$. This fact might be an additional limitation, depending on the application, to performing measurements of bioimpedance.

Nevertheless, AD5933 can perform measurements of loads below $1 \mathrm{k} \Omega$ when the output voltage is attenuated by an op-amp in inverting configuration as indicated in figure 3 .

\subsection{Analog front-end specifications}

The AFE must guarantee that the impedance measurement system is totally adapted to perform reliable four-electrode measurements of EBI according to the following specifications.

(i) The electrical safety of the patient must be ensured.

(ii) The dynamic range of measurement must be as expected in a human body.

(iii) The AFE must operate at any frequency supported by AD5933. 


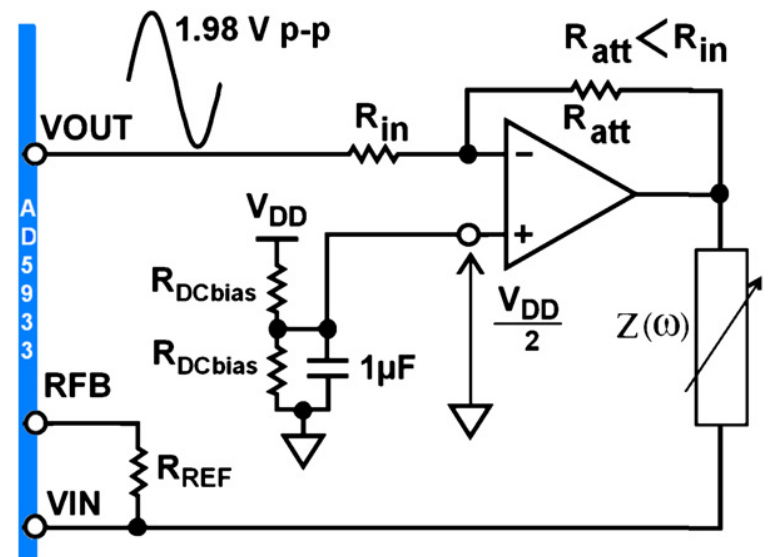

Figure 3. Attenuation circuit to adapt AD5933 for measurements of small impedance. The circuit proposed by Analog Devices in the datasheet of the evaluation board for AD5933. Note that $1.98 \mathrm{Vpp}$ is the output for AD5933 functioning in Range 1 operation mode. Note that the resistive values used for implementing the circuit are $1 \mathrm{k} \Omega$ and $4.13 \mathrm{k} \Omega$ for $R_{\text {att }}$ and $R_{\text {in }}$ respectively, and the op-amp was TL082.

In order to achieve the aforementioned requirements, besides having an excitation and sensing ports totally independent of each other, i.e. four-electrode measurement setup, the AFE must block the flow of dc current through the TUS while simultaneously ensuring that the value of the ac current complies with the safety regulations imposed by IEC-60601. All these must be provided by the AFE while keeping the signal input and output signals of the AD5933 circuit within operational levels, i.e. avoiding current or voltage saturation.

\subsection{System function and interfaces}

The AFE is an interface between AD5933 and the TUS. As such, it must have the proper input and output stages to seamlessly interconnect to each of them. For a better understanding of the following description, it is advised to follow the block diagram depicted in figure 4.

In short, we could consider the AFE as a combination of two voltage-to-current converters (V2CC), one in the direction from AD5933 to the TUS and another from the TUS to AD5933.

Since AD5933 applies voltage and expects a current flowing into its RFB input, the AFE interfacing with AD5933 has a voltage input and a current output. The current source output expressly generates the current resulting from the ratio of $V_{\text {out }}$ and the impedance of the TUS, which is the current expected by AD5933 at the RFB input.

At the TUS side, the AFE has a current source as output while the input is a differential voltage measurement channel. The current source excites the TUS with an adjustable current. In this case, a current of $350 \mu \mathrm{A}$ rms has been selected, thus fully complying with IEC-60601 for measurements above $3.5 \mathrm{kHz}$.

In essence, the AFE operation can be described as follows: after the removal of the dc bias component from the voltage output of AD5933 with a high-pass filter at the input of the first V2CC. The ac voltage from $V_{\text {out }}$ drives a voltage-controlled current source (VCCS) injecting an ac current $I_{\text {out }}$ into the TUS. Note that $I_{\text {out }}$ is directly proportional to $V_{\text {out }}$. The ac current $I_{\text {out }}$ causes a voltage drop at the TUS, which is sensed by the second V2CC and, since the voltage drop at the TUS drives the second $\mathrm{V} 2 \mathrm{CC}$, an ac current proportional to the voltage drop in the 


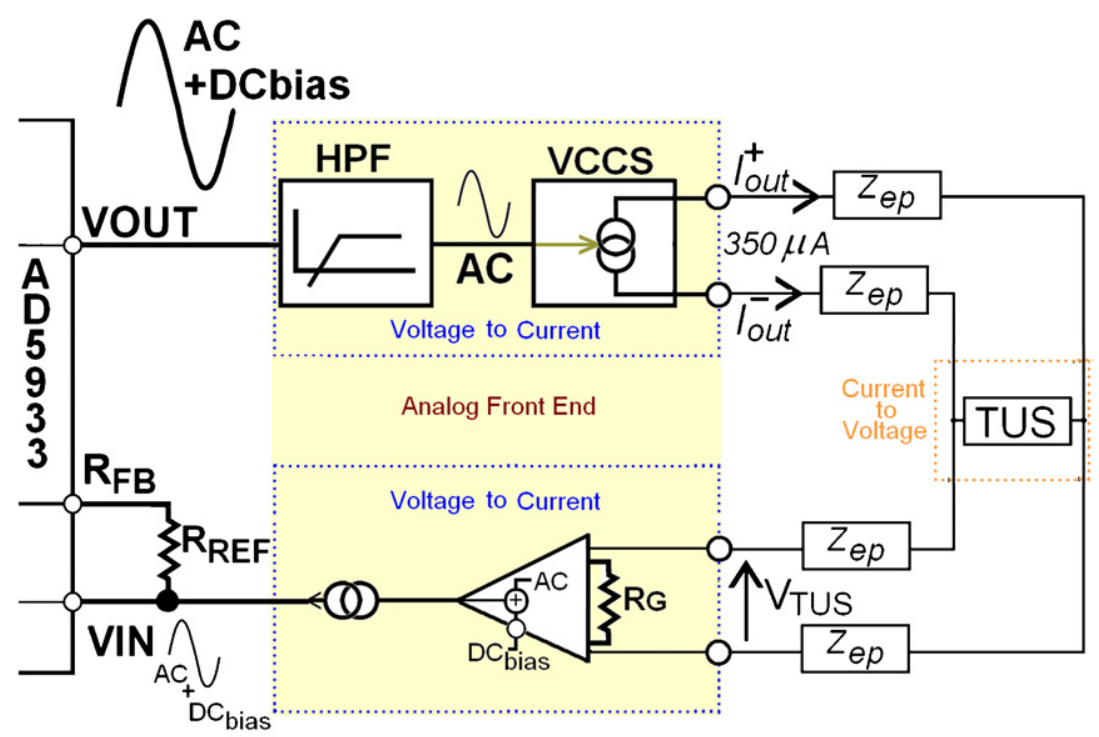

Figure 4. Functional block diagram of the proposed and implemented analog front-end.

TUS is generated. Finally, a dc component is added to the ac current generated. This added $\mathrm{dc}$ component is equivalent to $\mathrm{dc}_{\text {bias }}$ originally removed from $V_{\text {out }}$. Note that the total gain introduced by the cascade combination of both $\mathrm{V} 2 \mathrm{CC}$ together with the resistor $R_{\mathrm{REF}}$ at the input RFB sets the upper limit of the load dynamic range:

$$
V_{\text {DAC_max }} \geqslant R_{\text {REF }} \times g_{m 2} \times g_{m 1} \times V_{\text {out }} \times Z_{\text {TUS_max }} .
$$

Even when carefully selecting the transconductance functions $g_{m 1}$ and $g_{m 2}$ of the V2CCs in (3), the total gain of the AFE can be set to 1. The AFE introduces a critical change in the impedance estimation process implemented by AD5933. Originally, AD5933 implemented the impedance estimation by performing the quotient of a voltage signal over a current signal, assigned to the reference signal and the measurement signal respectively. The AFE modifies such signal assignation in such a way that the operation performed by AD5933 is the quotient between a current signal over a voltage signal, i.e. amperes over volts, corresponding with the admittance of the measurement load instead. This issue has to be considered by the software application when handling the estimated immitance data:

$$
\begin{aligned}
\frac{\text { REFERENCE SIGNAL }}{\text { MEASUREMENT SIGNAL }} & =\frac{V_{\mathrm{OUT}}(\omega)}{I_{\mathrm{IN}}(\omega)}=\frac{I_{\mathrm{load}}(\omega) / g_{m 1}}{g_{m 2} \times I_{\mathrm{load}}(\omega) \times Z(\omega)} \\
& =\frac{1}{Z(\omega)} \times \frac{1}{g_{m 2} \times g_{m 1}}=Y(\omega) .
\end{aligned}
$$

\section{Performance results}

\subsection{Accuracy}

The performance of the EBI measurement system can be observed in figures 5 and 6 . Figure 5 contains the measurement error obtained at $10 \mathrm{kHz}$ for measurements of resistive loads with both measurement arrangements, as follows. 


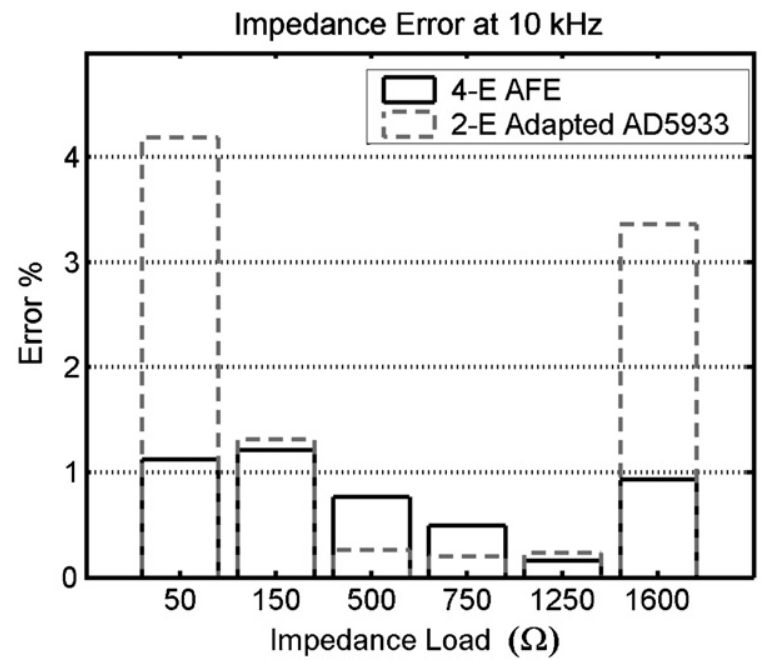

Figure 5. Impedance measurement error for measurements at $10 \mathrm{kHz}$ with both measurement arrangements. Note that the calibration has been done with a resistive load of $1000 \Omega$.

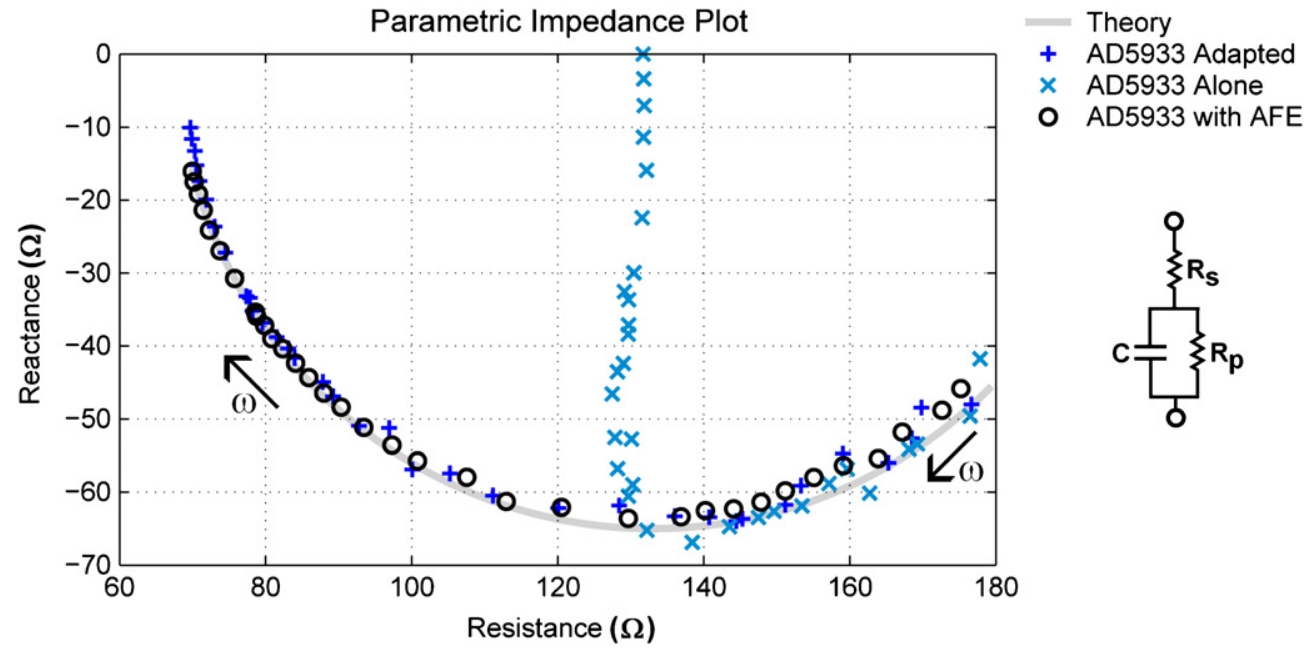

Figure 6. Parametric impedance plot of theoretical and measured values of a series $2 \mathrm{R}-1 \mathrm{C}$ circuit. $R_{\mathrm{S}}=68 \Omega, R_{\mathrm{p}}=130 \Omega$ and $C=100 \mathrm{nF}$. Frequency range: $5-100 \mathrm{kHz}$. Note that since the TUS used in this measurement is an electrical phantom, there is no electrode polarization impedance present, $Z_{\mathrm{EP}}$.

(i) Four-electrode setup using the AFE. The bar is plotted with discontinuous trace.

(ii) Two-electrode setup using the attenuation circuit described in figure 3. The bar is plotted with solid trace.

In general, the measurement error is kept very low for most of the impedance range, especially for values near the calibration value, $1000 \Omega$.

In figure 6, the parametric plot reactance versus resistance contains the impedance values of a $2 \mathrm{R}-1 \mathrm{C}$ series model. For comparison, the measurements obtained with AFE, without 


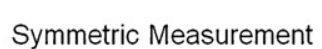

(a)

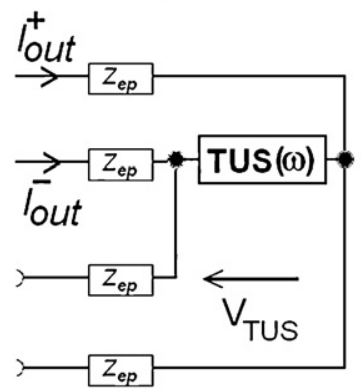

Asymmetric Measurement

(b)

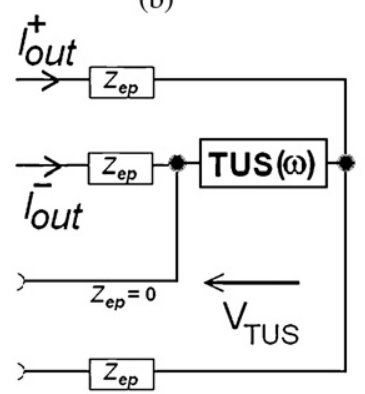

Electric Circuit for $Z_{e p}$

(c)

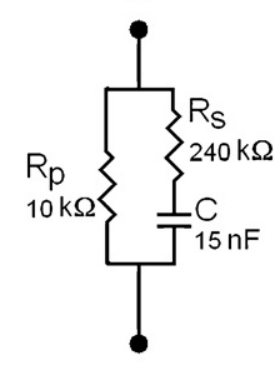

Figure 7. A lead imbalance experiment to test the proper implementation of the four-electrode method.

AFE and with the attenuation circuit are plotted together with the theoretical value. Note that the electrical series circuit is the measurement load as such, and therefore there is no electrode polarization impedance of any kind present in these measurements.

It is easy to note, in the impedance plots in figure 6, the remarkable agreement between the theoretical values and the measurement done with the AFE, especially at high frequencies. Note that the frequency range of the measurement is $5-100 \mathrm{kHz}$, increasing clockwise as indicated in the plots.

In figure 6, it is also possible to observe that the measurements performed with AD5933 and the attenuator circuit do not match the theoretical values as well as those with the AFE.

Another significant result to remark is the asymptotic behavior of the measurements performed with AD5933 alone. These measurements apparently indicate the lower limit of the impedance measurement range.

\subsection{Electrode polarization avoidance}

The effect of electrodes has been modeled by adding impedances to the measurement leads. The goal pursued with the addition of these impedances is to simulate the effect of the electrode polarization impedance $Z_{\mathrm{ep}}$. The electrode model used for this experiment is depicted in figure 7(c). Impedance measurements have been done with two different setups: symmetric and asymmetric, as depicted in figures 7(a) and (b) respectively, and the results of the corresponding measurements are plotted in the impedance parametric plot in figure 8 . The plot contains the value of the measurements, dotted trace and discontinuous trace for the symmetric case and the asymmetric case, respectively, and for comparison the theoretical value of the measurement load is plotted with the continuous trace.

\section{Discussion}

\subsection{On the application of AD5933 for electrical biomedical impedance}

The reported measurements strongly indicate that the AD5933 impedance converter by itself cannot be used for any application EBIS where proper electrical characterization is needed. This result was expected, since AD5933 performs two-electrode measurements only. 


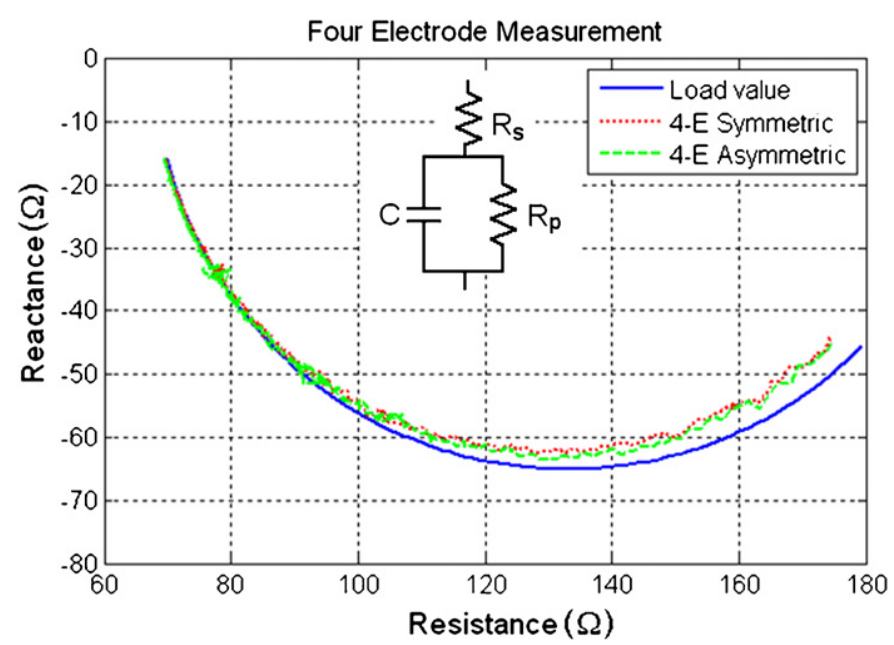

Figure 8. The parametric impedance plot of a series $2 \mathrm{R}-1 \mathrm{C}$ circuit. $R_{\mathrm{S}}=68 \Omega, R_{\mathrm{p}}=130 \Omega$ and $C=100 \mathrm{nF}$, with impedance in leads modeling the effect of electrodes. Frequency range: $5-100 \mathrm{kHz}$. Note that the value for the capacitor in the theoretical calculations is the nominal value, while the value for the resistors $R_{\mathrm{S}}$ and $R_{\mathrm{p}}$ is the value measured at measured dc.

In addition to this limitation, the excitation signal generated by AD5933 is not dc free and the signal is injected by a voltage source. An important consequence of the voltage excitation is that the current through the load is dependent on the impedance value of the load as well as on the electrode polarization impedance. Since the value of the working load, mostly the electrode polarization impedance, can change with time, the current through the tissue can exceed the limit imposed by IEC-60601.

Another important limitation regarding the voltage source of AD5933 is that its minimum output impedance, $R_{\text {out }}$, is $200 \Omega$. Such a value is very high for a voltage source, especially when the working load can be very small, as in electrical bioimpedance applications.

Moreover, apparently AD5933 cannot measure loads smaller than $130 \Omega$ approximately. See figure 6. This lower limit is related to the voltage source, $R_{\text {out }}$ of $200 \Omega$ and $5.8 \mathrm{~mA} \mathrm{Vpp}$ of the ac output current. With a maximum current of $5.8 \mathrm{~mA}$ and a voltage source providing around $1.9 \mathrm{Vpp}$, the minimum load is approximately $330 \Omega$, including $200 \Omega$ of $R_{\text {out }}$. This might not be a limitation in practice, since in a bioimpedance application the presence of the electrodes will increase the value of the working load.

\subsection{Frequency performance with a whole single dispersion capacitive system}

The measurements obtained with the proposed measurement system and the theoretical values agree significantly well in the whole frequency range. In this frequency range, we should not expect large parasitic effects in RC dummy circuits. Nevertheless, in a wearable application the measurement scenario is much more hostile, and the existence of parasitic capacitances might ruin the measurements. AD5933 has a built-in function for calibration, but at this point it is unknown to what extent such a feature will contribute to minimizing this type of negative effect.

\subsection{Accuracy performance and load dependence}

The reported experimental results indicate that the introduction of an external AFE does not significantly worsen AD5933. As expected, the results suggest that the best performance 
is obtained when the measurement load approximates to the calibration load. In a typical application of electrical bioimpedance, the target load will have a dynamic range much smaller than the impedance range considered in these tests. Therefore it is most likely that the impedance measurement system will be able to keep, for each specific application, the high accuracy reported in figure 5 .

\subsection{Four-electrode setup}

The four-electrode technique is successfully implemented with the AFE, as the measurements with simulated electrodes and particularly the asymmetric test show. The only source of concern is the observed deviation of both measurements at low frequencies from the theoretical value. This deviation may be due to the fact that the theoretical value for the circuit is calculated with the nominal value and not with the real value of the test components. The circuit overcomes the intrinsic limitation of the two-electrode structure of the AD5933 device. Note that measurements with the original two-electrode setup of AD5933 would have produced impedance values in the range of several $\mathrm{k} \Omega$, including the impedance of the electrode equivalent model (Ferreira and Sanchez 2007).

\subsection{Limitations}

The only foreseen limitation for this type of device is the upper limit frequency. A maximum high frequency of $100 \mathrm{kHz}$ is not enough for certain EBI applications, especially when the purpose of the measurement is to characterize the full beta dispersion. In the datasheets provided by Analog Devices, the option to measure above $100 \mathrm{kHz}$ is considered. We have not tested the performance of AD5933 above the recommended frequency range in this work because the objective of the work was to adapt AD5933 for EBI measurements.

Regarding safety issues of the AFE due to the excitation current, the implemented solution as such can be used to measure EBI at frequencies down to $3.5 \mathrm{kHz}$, complying with IEC60601. Simply by adjusting the transconductance of the first VCCS in the AFE, the output current can be adjusted to perform measurements of EBI at lower frequencies.

\section{Conclusions}

The analog front-end proposed in this paper complies with the initial requirements. It implements a complete four-electrode measurement system, and completely adapts AD5933 for electrical bioimpedance measurements. This achievement is obtained by the addition of very few ICs, in essence only two, and a few passive components. This simple analog frontend, in combination with the unique system-on-chip impedance spectrometer, reduces the size and complexity of the electronics of an EBI measurement system.

The development of wearable home-monitoring devices can benefit to a very large extent from such reduction in size and complexity, while it allows the implementation of EBI measurement systems with target sizes similar to mobile phones or even watches. The availability of such minimal monitoring devices would contribute substantially to spreading the use of EBI measurement for home monitoring and wearable applications.

\section{References}

Aberg P et al 2005 Non-invasive and microinvasive electrical impedance spectra of skin cancer-a comparison between two techniques Skin Res. Technol. $11281-6$ 
Amft $\mathrm{O}$ and Habetha J 2007 Smart Textiles for Medicine and Healthcare. Materials, Systems and Applications ed L Van Langenhove (Cambridge, UK: Woodhead Publishing) pp 275-301

Caduff A et al 2006 Non-invasive glucose monitoring in patients with diabetes: a novel system based on impedance spectroscopy Biosens. Bioelectron. 22 598-604

Ferreira J and Sanchez J J 2007 Electrical bioimpedance measurement system for limb edema monitoring Master Thesis School of Engineering, University College of Borås p 104

Hännikäinen J, Vuorela T and Vanhala J 2007 Physiological measurements in smart clothing: a case study of total body water estimation with bioimpedance Trans. Inst. Meas. Control 29 337-54

Medrano G et al 2007 Bioimpedance spectroscopy with textile electrodes for a continuous monitoring application 4th Int. Workshop on Wearable and Implantable Body Sensor Networks (BSN 2007) pp 23-8

Moissl U M et al 2006 Body fluid volume determination via body composition spectroscopy in health and disease Physiol. Meas. 27 921-33

Pacelli M, Loriga G, Taccini N and Paradiso R 2006 Sensing fabrics for monitoring physiological and biomechanical variables: e-textile solutions Medical Devices and Biosensors, 2006. 3rd IEEE/EMBS Int. Summer School pp 1-4

Pallàs-Areny R and Webster J G 2001 Sensors and Signal Conditioning (New York: Wiley-Interscience)

Paradiso R and De Rossi D 2006 Advances in textile technologies for unobtrusive monitoring of vital parameters and movements Engineering in Medicine and Biology Society, 2006. EMBS '06. 28th Annual Int. Conf. IEEE (New York) pp 392-5

Paradiso R, Loriga G and Taccini N 2004 Wearable health care system for vital signs monitoring MEDICON 2004 (Naples)

Scheffler M, Hirt E and Caduff A 2003 Wrist-wearable medical devices: technologies and applications Med. Dev. Technol. 14 26-30

Seppä V P, Väisänen J, Kauppinen P K, Malmivuo J and Hyttinen J 2007 Measuring respirational parameters with a wearable bioimpedance device ICEBI (Graz: Springer)

Van Langenhove L 2007 Smart Textiles for Medicine and Healthcare. Materials, Systems and Applications (Cambridge, UK: Woodhead Publishing)

Vuorela T, Seppä V P, Vanhala J and Hyttinen J 2007 Wireless measurement system for bioimpedance and ECG 12th Int. Conf. on Electrical Bio-Impedance ed H Scharfetter and R Merwa (Graz: Springer) 